\title{
Mapping Algorithm Design and Maturity Model Construction of Online Learning Process Goals
}

\author{
https://doi.org/10.3991/ijet.v14.i04.10133 \\ Yang Hong $\left({ }^{\bowtie}\right)$, Zhou Xinyi \\ Chengdu Normal University, Chengdu, China \\ 20960567 eqq.com
}

\begin{abstract}
In order to solve the problems of resource storage, resource delivery delay and other issues in the online learning process, it is studied and analyzed from the perspective of process goals. Firstly, a measurable e-learning process capability maturity model (EPCMM) is established. Then, based on the analysis of workflows in EPCMM's five process areas, a mapping relationship between major activities and key objectives is established in the process area. Finally, the mapping algorithm of online learning process features to process goals and the mapping algorithm of online learning metrics to process features are given. The practical results show that compared with the classical algorithm, this algorithm can effectively reduce the average transmission delay of online learning resources and improve the load balance of the system.
\end{abstract}

Keywords-Online learning, mapping algorithm, process, model

\section{Introduction}

The advancement of network technology and the characteristics of media have given learning a new carrier. A standardized online learning concept is becoming a new thing that enterprises, educational institutions and government agencies take seriously. The global trend and commercialization of online learning will be an important educational change in the $21 \mathrm{st}$ century. Whether online learning has achieved success or whether educational investment has achieved the desired educational benefits requires evaluation. Evaluation is the basis for conducting online teaching activities. With the increasing popularity of multimedia technology and the Internet, information technology is changing the way people produce, work, live and learn in human society, and this change is increasing. In recent years, the development of the network industry has deeply affected people's lives from all levels. In today's world, online media has become a mass media that can't be ignored and is becoming more and more important.

With the development of modern information technology, it has had a profound impact on the way people learn, and this has led to tremendous changes in the field of education and teaching. Today, the form of education has extended from traditional stage school education to on-the-job training and lifelong education, and the demand for learning has reached an unprecedented level. In the past, various forms of 
traditional education have been unable to meet the rapidly updated requirements of social knowledge and skills. As online learning becomes more and more accepted by the public, many companies specializing in online learning have emerged. The services provided are basically divided into three categories: the first category provides a technology learning management platform, the second category provides content, and the third category provides specialized services. Generally speaking, online learning evaluation belongs to the sub-category of educational evaluation, and has the three meanings of educational evaluation, namely value judgment, evaluation development and reference standards. However, online learning has its own unique personality compared with traditional learning. For example, the separation of teaching and learning activities in time and space, the realization of teaching requires a reliable and secure network transmission system, and the learning of learners is mainly self-learning. Therefore, online learning evaluation also shows its unique characteristics.

Online learning is not simply a copy of the traditional full-time education model, which relies on high-speed information technology and networks. Traditional textbooks and classroom face-to-face teaching have been replaced by autonomous learning models that combine unbounded and shared online learning resources with self-study and face-to-face tutoring. The traditional teaching model centered on textbooks and teachers is being challenged as never before. Online learning is a new education mode, which runs through the education concept of cultivating people with comprehensive development and advocates new concepts of learning, teaching, and management. The learning and teaching activities of online learning are mainly realized with the Internet. It makes full use of the learning environment provided by modern information technology with new communication mechanism and rich resources to realize a new learning method. Whether online learning has achieved results and whether educational investment has achieved the desired educational benefits requires an evaluation. Evaluation is the basis for conducting online teaching activities.

\section{$2 \quad$ Literature Review}

Acostagallegos et al. (2015) pointed out in the study that there are four components in the analysis of learning essence, namely learning subject, learning conditions, learning outcomes and learning process. Human learning is a social phenomenon that is constrained by the development of social history and the physical and mental characteristics of people. Learning is the means by which individuals adapt to the environment. Learners interact with the environment and constantly change their behaviors to adapt to survival and acquire their own needs [1]. Wilcox et al. (2015) pointed out in the study that learning is the activity of acquiring skills in terms of purpose and result. From the perspective of pathways and processes, the objective things of perception, reflection and change are all learning. From the content and the harvest, learning is an activity that acquires information knowledge, forms skills, improves intelligence, and optimizes quality. As long as people live in the 
environment, as long as they perceive, think and change the environment, they are learning [2]. Wren et al. (2014) pointed out in the study that learning is the process of obtaining information, processing information and applying information. Acquiring information is the prerequisite, processing information is the key, and applying information is the purpose. The process of processing information includes identifying the attributes of the information, the function and value of developing the information [3]. Bozzano et al. (2014) pointed out that learning is a process of planning, purposefully acquiring, processing, and applying information to form skills, improve intelligence, and improve diathesis [4]. Ferralis et al. (2015) proposed an Agent-based model in the study that combines learning models, balanced scorecards, and option pricing to provide a dynamic, flexible framework for evaluating the implementation of online learning projects [5]. Bartocci et al. (2015) pointed out in the study that ordinary online learning systems focus on the management of learning objects and learning outcomes, but it ignores the learning process. Workflow-based instructional activities can help support, manage, and monitor the learning process. In their papers, the application of work communication learning in online learning environment and the construction idea of teaching environment based on workflow management system are discussed [6]. Duc et al. (2015) pointed out in the study that the evaluation of online learners' reading ability should be based on the analysis of reading materials, the evaluation of learners themselves, the evaluation of reading details and the reading scores. In this way, the potential of learners can be stimulated to the maximum extent and the teaching can be promoted [7]. Sentelhas et al. (2015) introduced Internet-based distance learning evaluation standards in research. This standard includes architecture, curriculum development, teaching and learning, curriculum structure, student support system, teacher support system, evaluation system. These aspects are further refined into several necessary core indicator items and several non-essential optional indicator items. At the same time, the report also introduced a case study on the evaluation of the college's online courses with this standard [8].

The first part introduces the research background, explains the importance and necessity of this topic. The second part summarizes the research on E-learning. The third part constructs the E-learning Process Capability Maturity Model, introduces the key indicators of E-learning process domain, and describes the mapping relationship and algorithm between E-learning process and measurement. The fourth part introduces E-learning Process Capability Maturity Model and describes the characteristics of this model. The fifth part gives the conclusion.

This study introduces the process management evaluation framework into the field of E-learning, and its feasibility and practical significance are obvious. The proposed EPCMM model can be regarded as an exploratory step in this research direction. However, there are still some limitations in this study and no perfect model has been proposed. Therefore, the complete E-learning process evaluation system still needs further research and development. 


\section{$3 \quad$ Methodology}

\subsection{Construction of online learning process maturity model}

As a process-based online learning maturity model, E-learning maturity model (EMM) plays an active role in evaluating the organization's ability and level of online learning activities. However, the EMM model itself uses the method of color block labeling to indicate the maturity and level of the organization's activities in the various process areas of online learning. It has inherent defects in effectively controlling and improving the online learning process. Therefore, the analysis and evaluation methods of high maturity ability in the Capability Maturity Model (CMM) are used for reference, and a new online learning process capability maturity model is established with the quantitative method, which is EPCMM.

The key of the EMM dimension concept is to embody the overall phased capabilities of the organization, rather than a gradual horizontal measurement model. It describes the capabilities of the process from a collaborative point of view. For an organization, if it develops in all dimensions of all processes, it will be better than those that do not. Higher level capabilities will not produce the desired results without the support of lower level capabilities. Without the support of higher-level capabilities, lower-level capabilities become very special, unsustainable, and unable to respond to the needs of changing organizations and learners. In the process of online learning, the relationship between abilities at all levels can be studied, as shown in Figure 1:

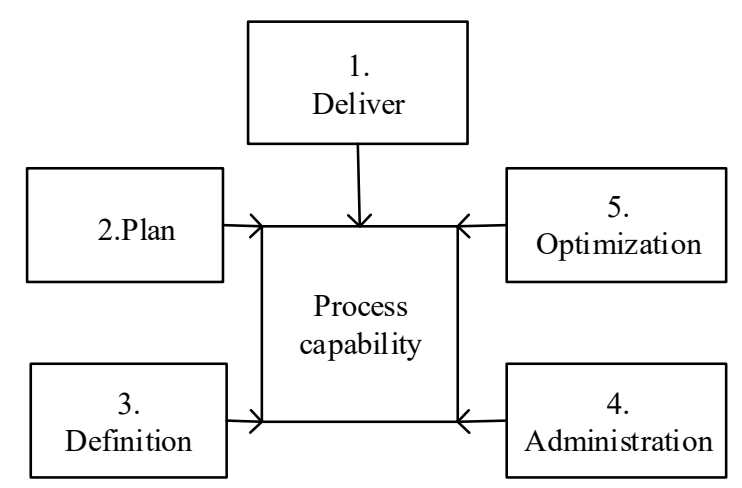

Fig. 1. Measurement dimension of online learning process

Take Marshall's model as a prototype and refer to all the key indicators in the five process domains of EMM. First, quantify each process activity. The previous section discusses the metrics of the online learning process, including five dimensions of delivery, planning, definition, management, and optimization. Estimated value $\mathrm{V}_{\mathrm{i}}$ of each process behavior is respectively set on the above five dimensions, $i=\{1,2,3,4$, 5 . And the estimated value is set according to the four performances: insufficient, 0 points; partially sufficient, 2 points; most sufficient, 4 points; fully adequate, 6 points; 
that is, $\mathrm{V}=\{0,2,4,6\}$. Then, the estimated value of the five dimensions are averaged by weighting, and the obtained estimated value of the process behavior is a, that is:

$$
a=\sum_{i=1}^{5} W_{i} * V_{i}
$$

In the above formula, $\mathrm{W}$ represents the weight of the process behavior in each dimension. For the sake of simplicity, it is assumed that the influence of a process behavior in five dimensions is equal, so all the above five weights are set to 0.2. Taking the learning process in Marshall's model as an example, there are 10 process behaviors, and the estimated value of each process behavior is obtained by weighted average of its 5 dimensions. If all processes are full scores in each of their dimensions, then all values of a are 6 and the total score is 60 points. In this way, the full score of the five process categories in the Marshall model can be calculated. The learning process is 60 points; the development process is 36 points; the collaboration and support process are 66 points; the evaluation process is 42 points; and the organizational process is 54 points.

Next, the EPCMM process metamodel is built. The model is a two-dimensional structure, as shown in Figure 2:

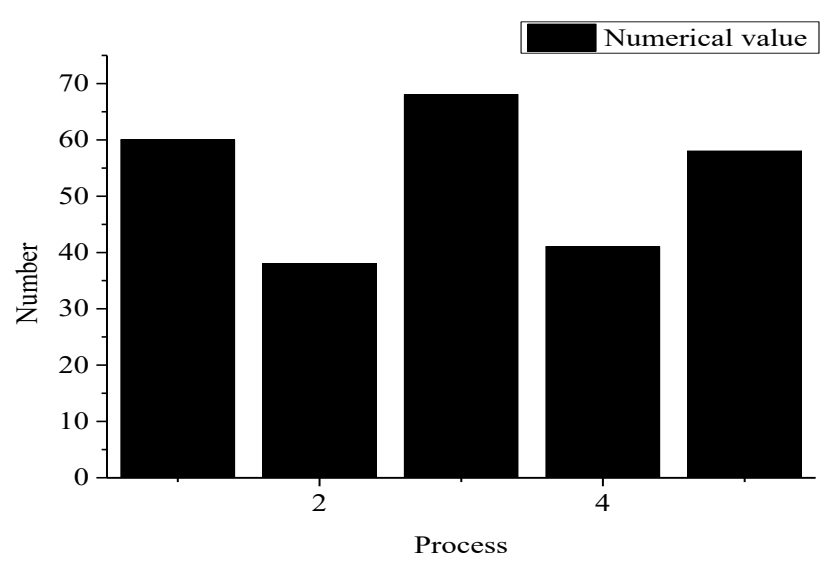

Fig. 2. EPCMM Process metamodel

The above diagram represents the structural framework for development, learning, cooperation, evaluation, and organization. The process categories defined in each EPCMM are evaluated according to Marshall's model, which shows that all process behaviors are rated as full scores. The above process metamodel can be expressed as a continuous model or as a phased model. As a continuous model, the process behavior on each process category is changeable, and the estimated value of its process categories reflected in the model is also variable. As a phased model, when the process behavior of each process category is fixed at a certain stage, the model shows 
the stage level and ability of an educational institution to implement online learning. In actual work, each online learning organization can define its own online learning process metamodel according to its own organizational standards.

The EPCMM phased model provides a predefined roadmap for organizationallevel improvements based on relationships at the process level, represented by a series of stages called maturity. Each maturity level covers all online learning process domains, each of which is described by key metrics that meet its objectives, the metrics are determined by values in each dimension (Equation 1). Process improvement progress is achieved through all process domain objectives in a particular maturity level. The EPCMM's phased model is defined as 6 maturity levels (MLs). ML0: initial level; ML1: delivery level; ML2: planning level; ML3: definition level; ML4: management level; ML5: optimization level. The definition of maturity level of online learning process is shown in table 1.

The maturity level is a stepped model, as shown in Figure 3.

Table 1. Maturity level definition

\begin{tabular}{|c|l|}
\hline Maturity level & \multicolumn{1}{c|}{ Describe } \\
\hline 5 & Continuous improvement in all aspects of the online learning process. \\
\hline 4 & Ensuring online learning resources and quality of learning. \\
\hline 3 & Developing and supporting the definition process for online learning. \\
\hline 2 & An online learning project has a clear and measurable goal. \\
\hline 1 & Create an online learning process and produce process results. \\
\hline 0 & No process is defined. \\
\hline
\end{tabular}
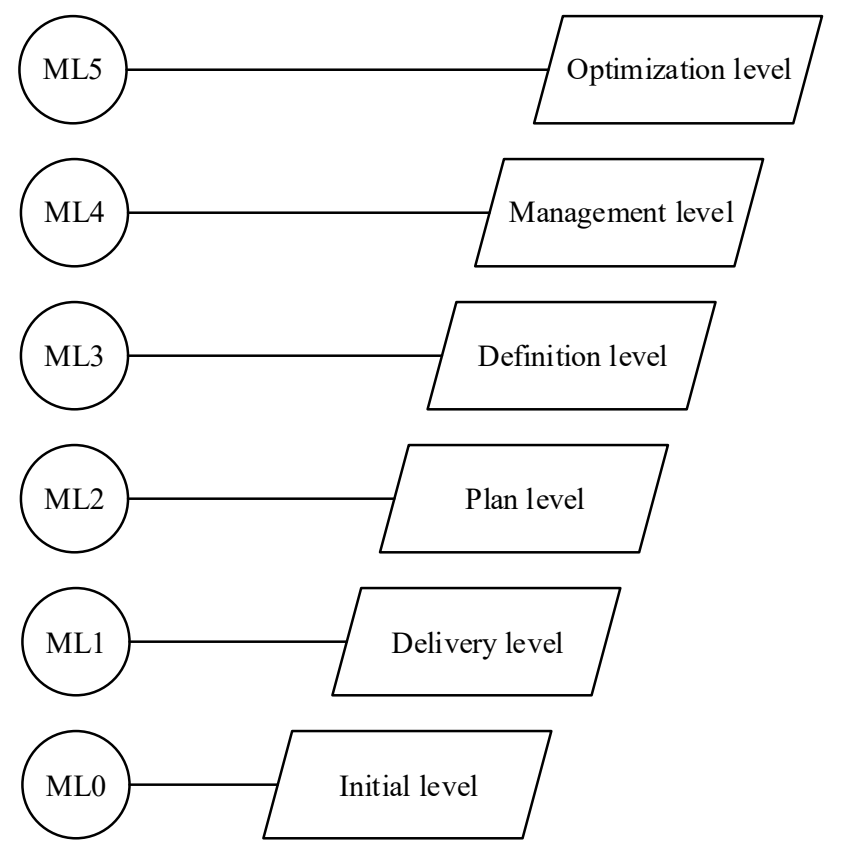

Fig. 3. Phase model 
The online learning phased model has two advantages: first, the phased model provides a process platform for supporting the organization's online learning process improvement. For organizations looking to improve the maturity of the online learning process, the phased model provides a clear, effective and progressive approach to development. In the six online learning maturity levels of the organization described in the phased model, each time a level crossing is achieved, the organization is committed to solving a problem in a certain dimension in all online learning process domains; second, the staged model can define an online learning process maturity level for the organization, facilitating horizontal comparison across organizations. The expected range and application of the process domain is very clear in the online learning maturity level based on the phased model.

There are two disadvantages in the online learning phased model: first, the phased model divides the online learning process domain into six levels. In general, an organization must meet all of the process domains of the level and its low level in order to reach a certain level, thus lacking flexibility; second, each level of the staged model has more process improvement work that needs to be performed at the same time, so the workload is large and the cost is also large.

Online learning continuous models are not clearly defined in the order in which improvements are made. There are no discrete phases associated with organizationallevel maturity, nor a definition of the overall ability classification of the organization, but a different capability distribution CL for any process. The continuous model of online learning is defined as six capability levels (CLs). CL0: incomplete level; CL1: executed level; CL2: planning level; CL3: definition level; CL4: management level; CL5: optimization level. Organizations can choose to perform the appropriate processes as needed, without being limited by the stage model's limitations on the process domain. Like the online learning phased model, the online learning continuous model also has a process domain with time. Similarly, each process domain is described by key metrics that meet its objectives, and its metrics are determined by values in each dimension (Equation 1). The difference is that the practice of the process domain in the online learning continuous model is the way to support the improvement of a single process domain. In a continuous evaluation, each process domain is rated by capability level. The different process domains of the two organizations are rated as different capability levels. It is described with key metrics that meet its objectives, and its metric is determined by the values in each dimension (Equation 1). It is a superimposed model and can't be gapped between levels.

The online learning continuous model also has the following two advantages: first, the online learning continuous model provides users with greater freedom to improve the online learning process. It allows users to select the order of process improvement activities based on the business purpose of the organization. In a continuous model, the user can choose to define the order of the process domains that are more suitable for their business environment; second, based on the continuous model, the organization's online learning process is evaluated, and the organization can make vertical comparisons with previous capabilities in the same process domain. In a continuous model, multiple capability levels can be defined for different process domains, which can enhance the understanding of strengths and weaknesses in 
process improvement. The continuous model also has two shortcomings: first, since the online learning continuous model does not specify the order of the process domains, the organization's online learning process improvement requires the guidance of the online learning process improvement experts to determine the process domains and improvement priorities that the organization needs to improve; second, although the organization has made process improvements with the online learning continuous model, it is difficult to compare the inter-organizational process capabilities with other organizations.

\subsection{Key indicators on the online learning process domain}

In the learning process domain, a total of seven process indicators are defined;

The practical activities on key indicator 1 need to meet the following requirements: learning outcomes are primarily derived from educational intent or learning objectives, which clearly describe the learning content, the actions or performances to be taken, and the evaluation method. High-quality learning objectives are clearly reflected in teaching methods and content, along with flexible and diverse teaching attitudes, learning processes and styles. A formal assessment is given, which protects the interests of the learner. Learning objectives are well documented, teaching strategies, ideals, values are defined, and the learning process is explored rather than just testing knowledge.

The practical activities on key indicator 2 need to meet the following requirements: in this process activity, its process capability is reflected in encouraging students to use various communication methods or channels to participate in communication between classmates and teaching staff. In fact, providing tools alone is not enough. It is also necessary to dynamically encourage and support the use of tools and participation in communication. It should provide students with information on how to access and use different communication channels. It should give students a clear explanation of why channels or models are included in the curriculum and how they will help achieve learning goals. In particular, when many students are not familiar with online learning, it is necessary for them to get clear information on how to use communication channels effectively and appropriately.

The practical activities on key indicator 3 need to meet the following requirements: the ability of students to effectively carry out an activity is reflected in the skills of the learners they possess and the ability to effectively utilize the various sources of information and the technology provided by the institution. It can be assumed that students have some level of technical ability and experience, but this does not mean that students are doing effective online learning. When designing instructional elements, it must consider how to ensure clear guidance to students and how technology should be used to support student learning. Technology and pedagogy must be clearly reflected in the curriculum and programs designed and provided.

The practical activities on key indicator 4 need to meet the following requirements: effective interaction requires careful planning and thoughtful management to ensure that responses to students meet their expectations while being clear. Under this requirement, the type of classification reaction is very effective for online learning 
environments with complex needs. It is also necessary to train students to use communication tools and provide strong technical support.

The practical activities on key indicator 5 need to meet the following requirements: provide a variety of communication channels, supplemented by a formal evaluation feedback process. Timely and useful feedback on policies should be required to improve students' relevant abilities, not just to meet current goals. Teaching staff should provide guidance and assistance for more effective feedback. Students receive feedback from teachers and other students that can be used to compare the gap between actual performance and expectations. Timely and constructive feedback affects student participation, curriculum performance and learning outcomes.

The practical activities on key indicator 6 need to meet the following requirements: link the content of the resource search and other information to the appropriate database by providing resources and guiding research to indicate where to find the appropriate reference material. Support the skills of using a variety of information to develop students' skills to effectively access useful resources. More comprehensive research skills should also be reflected in the assessment tasks and related feedback. The development of information literacy and skills should be reflected in the learning objectives. Provide teaching staff and students with templates, examples, training and support to use existing information resources to support student learning. Clear guidance and support on policy and intellectual property issues should be provided to teaching staff and students.

The practical activities on key indicator 7 need to meet the following requirements: teaching design should reflect diversity. Diversity encompasses coherent themes that involve accessibility and learning preferences. Inclusive support theory improves accessibility and all beneficial learning styles. This includes differences in the required respect ability and learning style. At the same time, it is required to respect values, guidelines, language factors, cultural and national traditions, and learners of special requirements. Inclusiveness also involves gender issues. In short, inclusive design benefits all learners.

\subsection{Mapping relationship and algorithm of online learning process and metrics}

Online learning organizational goals and objectives related to cost, quality or time can be mapped to appropriate online learning processes through quantitative indicators, so that online learning process objectives can be developed. In order to ensure the effectiveness of the measurement activities, a reasonable method must be used to select the metrics. Indicators are both a quantification of goals and a basis for process metrics. To achieve the goal of the evaluation process, a set of effective indicators should be used to measure process quality and performance.

Figure 4 shows the mapping relationship between the goals and characteristics of the online learning process. Here, based on this definition, the mapping relationship between the online learning process objectives and the process domain key indicators needs to be analyzed, so that the metrics about these key indicators can be effectively 


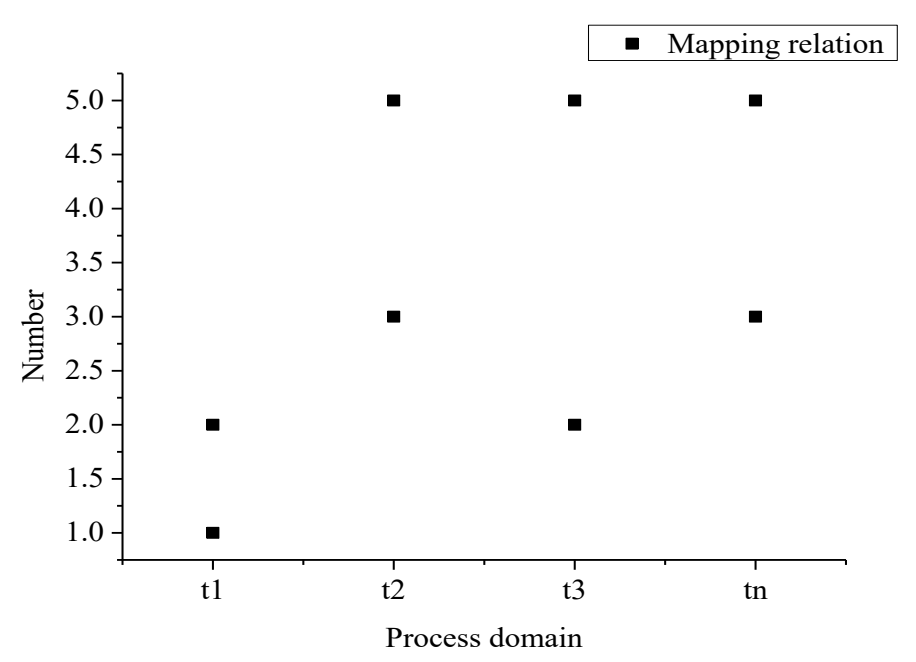

Fig. 4. Mapping relation function diagram

integrated into different processes. The online learning process goal is achieved by mapping key metrics to the appropriate online learning process. The determined organizational goals are measured by a range of key indicators. When the key indicators meet the requirements, the organizational goals can be achieved. The achievement of these key indicators is achieved by implementing activities in the relevant process domains.

The function of the online learning goal process is:

$$
\theta(p g)=P_{\text {Goal }}, P_{\text {Goal }} \subseteq P
$$

The mapping algorithm steps of the online learning process target are as follows: Mapping algorithm from online learning features to process objectives: Mtg

Input: any feature $\mathrm{t}$

Output: process / objective pair set (PnGT)

Steps:

PnGT $=\theta, \mathrm{P}=$ process ensemble $/ /$ initialization

Loop1:

Break if process set $\mathrm{P}=\theta$

$\mathrm{P}=\mathrm{P}-\mathrm{p} / /$ take process

Loop2:

Break if $\mathrm{P}=\theta$

If $\mathrm{t}$ belongs to $\mathrm{P}$

$\mathrm{PnGT}=\mathrm{PnGT}+<\mathrm{P}, \mathrm{Pgt}>$

End Loop 2

End Loop 1 
Any given process has one or more specific characteristics that can collect data. It is the metrics corresponding to these characteristics that can be used to measure process performance. In turn, the indicator measurement of the process performance can quantitatively measure and improve the quality characteristics. In any case, it is necessary to map a process goal with process activity and its indicator metrics. In online learning practices, workflow analysis provides an effective way for practitioners to effectively map process activities to process goals.

\section{$4 \quad$ Results}

EPCMM is an effective model for evaluating process capability maturity for organizations conducting online learning activities. Control methods can effectively help organizations monitor the implementation of the process. According to the process improvement guidelines and plans formulated by the organization, it is possible to effectively control a process so that the online learning process activities can be established at a stable level to achieve the organization's strategic goals. EPCMM staged model can be used to make horizontal comparison of maturity across organizations, while the EPCMM continuous model can be compared longitudinally against the previous capabilities of the organization on the same process domain.

The formal definition of online learning process modeling is based on software engineering. Accordingly, the definition of process metrics is combined with process definitions as an extension of the process definition. This approach better eliminates the ambiguity between the process definition and the process metric definition, making the implementation of the metric plan consistent with its definition. Based on the organization's standard online learning process, an online learning project executable process is generated. Based on the online learning process capability baseline, an online learning project plan control benchmark is established. At the same time, relevant responsible persons are assigned to the online learning process activities, and online learning process guidelines and criterions are established to ensure the effective implementation of the online learning process.

After establishing a series of workflows based on the EPCMM process model, it is necessary to ensure that the main activities in those workflows cover all the key indicators in each process domain of the model, with reference to the key indicators listed in the paper. At the same time, activities in the workflow on each particular process domain are necessary to ensure that key metrics are only mapped to this activity in a particular process domain.

\section{Conclusion}

The formal definition of online learning process modeling and the generation algorithm of online learning project execution process lay the foundation for the definition of the organization standard online learning process. It also provides an effective theoretical basis and practical method for developing automated tools that use online learning process definition and process control. The organization's online 
learning goals are measured by a set of key indicators. When the key indicators meet the requirements, the organizational goals can be achieved. The achievement of these key indicators is achieved by implementing activities in the relevant process domains. A mapping relationship between main activities and key indicators is established on the online learning process domain.

The online learning process capability baseline consists of a process eigenvalue and an upper bound deviation control limit. If the actual metric value at the time of the process execution falls within the upper and lower control limits, the process is stable. The Statistical Process Control (SPC) method can effectively help the organization to monitor the implementation of the process. According to the process improvement guidelines and plans formulated by the organization, the online learning process can be effectively controlled, and the online learning process activities can be established at a stable level to achieve the strategic goals of the organization. When the sample range is stable, the corresponding practice activities are included in the new online learning process improvement plan, and the key indicator list is refreshed. The current CL can serve as a reference point on the baseline of new online learning process capabilities.

\section{Reference}

[1] Acostagallegos, J. A., Kelly, J. D., Gepts, P. Prebreeding in common bean and use of genetic diversity from wild germplasm. Crop Science, 2015, vol. 47(3), pp. S-44-S-59.

[2] Wilcox, B. B., Robinson, M. S., Thomas, P. C., Hawke, B. R. Constraints on the depth and variability of the lunar regolith. Meteoritics \& Planetary Science, 2015, vol. 40(5), pp. 695-710. https://doi.org/10.1111/j.1945-5100.2005.tb00974.x

[3] Wren, T. A., Kalkwarf, H. J, Zemel, B. S., Lappe, J. M., Oberfield, S., Shepherd, J. A. Longitudinal tracking of dual-energy x-ray absorptiometry bone measures over 6 years in children and adolescents: persistence of low bone mass to maturity. Journal of Pediatrics, 2014, vol. 164(6), 1280-1285. https://doi.org/10.1016/j.jpeds.2013.12.040

[4] Bozzano, M., Cimatti, A., Katoen, J. P., Katsaros, P, Mokos, K., Nguyen, V. Y. Spacecraft early design validation using formal methods.Reliability Engineering \& System Safety, 2014, vol. 132(46), pp. 20-35. https://doi.org/10.1016/j.ress.2014.07.003

[5] Ferralis, N., Liu, Y., Bake, K. D., Pomerantz, A. E., Grossman, J. C. Direct correlation between aromatization of carbon-rich organic matter and its visible electronic absorption edge. Carbon, 2015, vol. 88(9), pp. 139-147. https://doi.org/10.1016/j.carbon.2015.02.075

[6] Bartocci, E. Bortolussi, L., Nenzi, L., Sanguinetti, G. System design of stochastic models using robustness of temporal properties. Theoretical Computer Science, 2015, vol. 587, pp. 3-25. https://doi.org/10.1016/j.tcs.2015.02.046

[7] Duc, G., Agrama, H. Bao, S., Berger, J., Bourion, V. Ron, A. M. D. Breeding annual grain legumes for sustainable agriculture: new methods to approach complex traits and target new cultivar ideotypes. Critical Reviews in Plant Sciences, 2015, vol. 34(1-3), pp. 381411. https://doi.org/10.1080/07352689.2014.898469

[8] Sentelhas, P. C., Battisti, R., Câmara, G. M. S., Farias, J. R. B., Hampf, A. C., Nendel, C. The soybean yield gap in brazil - magnitude, causes and possible solutions for sustainable production. Journal of Agricultural Science, 2015, vol. 153(8), pp. 1394-1411. https://doi.org/10.1017/S0021859615000313 


\section{Authors}

Yang Hong (corresponding author) is a lecturer Chengdu Normal University (20960567@qq.com)

Zhou Xinyi is a lecturer Chengdu Normal University (zhuo Xinyi@163.com)

Article submitted 04 November 2018. Resubmitted 03 January 2019. Final acceptance 20 January 2019. Final version published as submitted by the authors. 